1 Running Title: Blastocystis update

2

3

4

5 Title: Current status of Blastocystis: a personal view

6

7

C. Rune Stensvold ${ }^{a, *}$, C. Graham Clark ${ }^{b}$

8

9

10

11

12 a Statens Serum Institut, Copenhagen, Denmark

13

b London School of Hygiene and Tropical Medicine, London, UK

14

$15{ }^{*}$ Corresponding author: CR Stensvold - run@ssi.dk

16

17 
Abstract

Despite Blastocystis being one of the most widespread and prevalent

21 intestinal eukaryotes, its role in health and disease remains elusive. DNA-

22 based detection methods have led to a recognition that the organism is much

23 more common than previously thought, at least in some geographic regions

24 and some groups of individuals. Molecular methods have also enabled us to

25 start categorizing the vast genetic heterogeneity that exists among

26 Blastocystis isolates, wherein the key to potential differences in the clinical

27 outcome of Blastocystis carriage may lie.

28

In this review we summarize some of the recent developments and advances in Blastocystis research, including updates on diagnostic methods, molecular

31 epidemiology, genetic diversity, host specificity, clinical significance,

32 taxonomy, and genomics. As we are now in the microbiome era, we also

33 review some of the steps taken towards understanding the place of

34 Blastocystis in the intestinal microbiota.

35 
45 It is now over 100 years since Alexeieff [1] first described the intestinal eukaryote Blastocystis but, despite the efforts of numerous researchers (especially in recent years), there are still many unknowns surrounding this organism. Most important of these is whether Blastocystis causes disease in humans. For every report linking Blastocystis with gastrointestinal or other symptoms there is another that finds no such link. There are a number of

51 factors that have contributed to this apparent lack of progress and these will

52 form the basis of this review. We would like to warn the reader at this early 53 stage that we ourselves are convinced only that there are no definitive data 54 yet available to resolve this issue.

55

\section{TAXONOMY AND EVOLUTION}

57

58 In culture, Blastocystis is generally spherical with no obvious surface features.

59 When stained, the most common morphological form seen has a large central 60 vacuole of unknown function and the cytoplasm with all the organelles is

61 visible as a thin peripheral layer between the vacuole and the cell membrane

62 (Figure 1). While many morphological forms have been described, the

63 significance of most is unclear, the boundaries between them are not discrete, 64 and some may well represent degenerating forms [2]. We refer the reader to 65 earlier reviews for more details [3-5]. The life-cycle is typical of most gut 66 protists, with a resistant cyst form for transmission and a trophic form that 67 divides by binary fission. More complex and alternative life-cycles have been 
described (discussed in [5]) but in our opinion there is no conclusive evidence for anything other than this simple two-stage cycle.

Blastocystis has a complicated taxonomic history. It has been viewed as a fungus, a sporozoan and even the cyst of another organism at various points in its history, until 20 years ago [6] when it was finally placed among the Stramenopiles. This is one of the major groups of eukaryotes [7], but one that, to date, contains only a single other human-infective eukaryote, Pythium. Blastocystis has none of the typical features of a stramenopile, which is in part why identifying its correct relationships took so long. (1)

Since its classification as a Stramenopile further data have emerged regarding the closest relatives of Blastocystis. These turn out to be poorly known flagellated or ciliate-like organisms that live in vertebrate intestines. While most Stramenopiles are free-living and aerobes, Blastocystis and its relatives are gut-living and anaerobes, although they do have mitochondrionlike organelles (see later). Blastocystis is related specifically to the Proteromonadidae and Slopalinida [8], but these cannot be considered close relatives. However, it seems likely that the common ancestor of these groups of organisms was already living in a gut and an anaerobe.

The simple spherical morphology of Blastocystis mentioned above applies to

all members of this genus. This means that morphology is of no use in

1 defining species. Traditionally, Blastocystis species have been defined by the 2 identity of their host, with all human Blastocystis being assigned to 
93 Blastocystis hominis. However, even before DNA sequences identified

94 Blastocystis as a Stramenopile it had become clear that significant

95 heterogeneity existed among human Blastocystis. Using serology,

96 isoenzymes and karyotyping, human Blastocystis were being divided into

97 subgroups [4], and this picture of variation was reinforced by direct and

98 indirect DNA sequence analyses [9]. Subsequent data have only added to the

99 diversity and have refined our understanding of this genus.

100

101 Analyses of human Blastocystis by different researchers always resulted in

102 the detection of variation, but each group came up with its own nomenclature

103 for the groupings it identified. To resolve this confusion a consensus

104 terminology was agreed [9] and this classification of human Blastocystis into

105 numbered subtypes has simplified communication among workers in this field.

106 At the time of the consensus two things were clear: 1. that humans were host

107 to a number of distinct small subunit rRNA gene (SSU-rDNA)-based subtypes

108 of Blastocystis, and 2. that most of these subtypes were also found in other

109 mammalian or avian hosts. This meant the host-linked binomial species

110 names were untenable, as the same organism was being called by multiple

111 names. For example, one grouping of Blastocystis hominis proved to be

112 genetically indistinguishable from Blastocystis ratti; both are now known as

113 Blastocystis subtype 4 (ST4).

114

115 The current taxonomy of Blastocystis follows a distinct structure for mammal

116 and bird organisms compared to all others [10]. The mammalian/avian

117 Blastocystis are subdivided into seventeen subtypes (STs), nine of which 
118 (ST1-ST9) have been found in humans. There is host range overlap

119 observed for many of these organisms (Figure 2). Blastocystis from reptiles,

120 amphibia and invertebrates retain Linnean binomial names for the most part.

121 This is largely because little investigation of diversity and host range of these

122 Blastocystis has been undertaken to date and so the same impetus to change

123 the nomenclature has not existed. Whether a similar situation involving broad

124 host-range and large genetic diversity will be uncovered in those organisms

125 remains to be seen; it seems likely, and therefore the nomenclature of

126 Blastocystis in those hosts may require a similar solution.

3. GENETIC DIVERSITY AND HOST SPECIFICITY

130 Subtypes of Blastocystis are discrete and no intermediate variants have been

131 uncovered to date despite extensive sampling from around the world.

132 However, many host species remain to be sampled, so this picture may

133 change. Guidance on how and when to define a new subtype has been

134 published [11]. The recommendation is that a minimum of $5 \%$ sequence

135 divergence from the SSU-rDNA of known subtypes is required before defining

136 a new subtype is appropriate. One of the reasons for establishing this

137 boundary is that Blastocystis subtypes are often assigned based on the

138 sequence with the closest similarity in sequence database searches, without

139 taking into account the degree of similarity. So a sequence that actually

140 represents a new subtype may be assigned to an existing subtype. This

141 misattribution has been a problem in some existing cases, for example ST13,

142 as discussed in reference [10]. Unfortunately, information attached to entries 
143 in GenBank databases are rarely corrected and this can result in

144 misidentifications being propagated forward in the literature.

146 The $5 \%$ level of divergence to define a new subtype was chosen in part

147 because variation within subtypes can also be substantial, up to at least $3 \%$

148 [11]. Therefore a single 'outlier' sequence that appears to be distinct and 149 potentially a new subtype could eventually merge into an adjacent subtype as 150 more sequences become available. Only as more subtyping data accumulate 151 will the validity of this arbitrary threshold be tested. Note that $5 \%$ divergence is 152 the recommendation for establishing new subtypes, where sampling is likely

153 to be limited. The divergence between some existing subtypes (for example, 154 ST6 and ST9) is actually less than 5\%. However, sampling is sufficient to give 155 us confidence that these are indeed distinct lineages rather than variants of 156 the same subtype. In other words, $5 \%$ divergence has been chosen as quite a 157 stringent criterion and more data may lead to the revision of new subtype 158 definitions in the future..

160 As mentioned earlier, nine distinct subtypes have been found in humans

161 (Figure 2). However 95\% of human infections sampled belong to one of just 162 four of these subtypes (STs 1-4; [12]) and only one of the human subtypes 163 has not yet been found in another host: ST9 can claim (at present) to be 164 restricted to humans. The four most common STs in humans have also been 165 detected in other hosts. Most frequently these hosts are other primates, but 166 they have also been found in various hoofed mammals, rodents and even 167 birds [10]. Conversely, the rarer subtypes in humans (STs 5-8) are more 
168 commonly found in other hosts: ST5 in hoofed animals, STs 6 and 7 in birds,

169 and ST8 in non-human primates. It has been suggested that these rarer

170 subtypes in humans are of zoonotic origin and there is some evidence to

171 support this: ST8 has frequently been found in zookeepers that work with non-

172 human primates [13], and ST5 is prevalent in piggery workers in Australia

173 [14], for example. However, there is no reason to suspect that human

174 infections involving the common STs (STs 1-4) originate from non-human

175 sources except in rare cases.

176

177 Exposure to Blastocystis-infected animals alone is not sufficient to result in an

178 infection. For example, ST10 is very common in livestock [10] but is yet to be

179 reported in humans. This suggests that variables other than just body

180 temperature are determining the ability of Blastocystis to colonize the human

181 gut; the gut flora may have an impact, for example.

182

183 The degree of genetic diversity within subtypes is quite variable. ST3 is

184 probably the most diverse of the well-studied subtypes - varying by ca. $3 \%$ in

185 the SSU-rDNA sequences - while ST4 shows the least variation, especially in

186 humans [15]. Diversity in these subtypes has been further explored using a

187 multi-locus sequence typing approach based on variation in several regions of

188 the mitochondrion-like organelle's genome [15]. MLST data are not yet

189 published for other subtypes. How genetic variability within a subtype is

190 reflected in phenotypic and functional variability is as yet unclear. However,

191 differences in adhesion and drug resistance between strains of Blastocystis

192 ST7 have been reported [16]. 
194 Intra-subtype variation has provided further insight into host specificity. For

195 example, ST3 is common in both humans and non-human primates [13].

196 However, MLST analysis divided ST3 into four clades and almost all human

197 samples fell into only one of these clades [15]. Where this was not the case,

198 the individuals concerned had work exposure to non-human primates, again

199 suggesting zoonotic transmission had occurred [15]. It would be interesting to

200 know whether such host specificity exists between variants within other

201 subtypes that are found in a wide range of mammals and exhibit genetic

202 diversity, like ST10 for example [17].

203

204 MLST has the potential to provide insight into geographic aspects of genetic

205 variation as well. However, this could be confounded by the increasing

206 population mobility in today's world: geographic differences will be starting to

207 break down. To date, it is only subtyping that has provided evidence of

208 geographic differences in Blastocystis distribution. Specifically, it has become

209 clear that ST4 has a restricted distribution, being rare or absent in South

210 America, North Africa, and the Middle East, while being the second most

211 common subtype in Europe (summarized in [12]). The reasons for this are

212 obscure, but when combined with the relatively low genetic diversity of ST4 in

213 humans the evidence suggests that ST4 may only have entered the human

214 population relatively recently (perhaps in Europe) and is yet to spread around

215 the world [12]. ST4 is also found in other hosts [10], but there is no link

216 between these hosts and Europe. 


\section{DIAGNOSIS AND MOLECULAR CHARACTERIZATION}

221 For most parasites, both direct and indirect diagnostic methods have been

222 developed. Direct methods include those based on morphology (microscopy)

223 and detection of DNA (typically PCR) or antigens (IFA, antigen ELISA, etc.),

224 while indirect methods are based mainly on detection of antibodies [18]. While

225 the potential utility of serology in the indirect detection of Blastocystis

226 infections remains unclear, some studies have used serology to look for

227 quantitative differences in antibody responses between symptomatic and

228 asymptomatic individuals ([19-20]; see also below).

230 With regard to direct detection methods, the use of diverse diagnostic

231 modalities of varying sensitivity may very well have impaired attempts to

232 define the role of Blastocystis in health and disease [21-23]. Molecular

233 methods developed to detect Blastocystis in genomic DNA extracted directly

234 from fresh stool have highlighted the sensitivity shortcomings of diagnostic

235 methods such as the traditional 'ova and parasites' (O\&P) work-up (used to

236 detect cysts of protozoa and larvae and eggs of helminths), culture methods,

237 and permanent staining of fixed fecal smears [24-26].

238

239 Simple stains like Lugol's iodine can be used as a quick aid to the

240 identification of Blastocystis in fecal smears or concentrates; the organism is

241 otherwise difficult to differentiate from other structures seen in unstained

242 preparations due to the lack of diagnostic morphological features. Trichrome 
243 staining is one of several permanent stains used for detection of trophic forms

244 of protozoa in feces. Blastocystis stains characteristically with Trichrome, and

245 this method had a specificity and sensitivity of $100 \%$ and $82 \%$, respectively, in

246 a study by Stensvold et al. [24].

248 Despite being the primary diagnostic tool worldwide, the use of microscopy to 249 detect Blastocystis has limited utility in clinical microbiology laboratories and 250 in generating data for clinical and epidemiological purposes: 1) Microscopy of

251 fecal concentrates - the commonly applied O\&P method - has very low 252 sensitivity in detecting Blastocystis [24, 27]; 2) there is no consensus on the 253 importance of the cell numbers (see below) or the various morphological 254 forms reported; and 3) microscopy cannot distinguish between genetically 255 highly dissimilar organisms (STs), which may differ in their clinical 256 significance, a situation potentially similar to Entamoeba histolytica and 257 Entamoeba dispar. Nevertheless, there are situations in which microscopy 258 may serve a purpose, such as those aiming to verify the presence of 259 Blastocystis in various types of non-human samples, including those of 260 environmental and animal origin, to inform hypotheses on transmission. For 261 instance, a recent study used microscopy to identify Blastocystis in various 262 environmental samples, including food, water, and fomites [28]. 263

264 Xenic in vitro culture (XIVC) is defined as culture in the presence of an 265 undefined bacterial flora. Blastocystis can be grown and propagated xenically 266 in a variety of media $[29,30]$. Perhaps due to its simplicity and low cost, 267 Jones' medium has been popular for both detecting and maintaining 
268 Blastocystis; another medium often used for isolation is Robinson's [29], while

269 we have also used LYSGM (a variant of TYSGM-9; [31]) for propagation when

270 large numbers of cells are needed. XIVC as a diagnostic tool using Jones'

271 medium has a sensitivity ranging from $52 \%-79 \%$ compared with real-time

272 PCR assays [26, 32].

273

274 The diagnostic utility of Ag-ELISA and immunofluorescent antibody staining

275 methods for the detection of Blastocystis, including commercial kits such as

276 ParaFlor B (Boulder Diagnostics, Boulder, CO, USAa), coproELISA ${ }^{\text {TM }}$

277 Blastocystis (Savyon Diagnostics, Ashdod, Israel), and Blasto-Fluor

278 (Antibodies Inc., Davis, CA, USA), is as yet unclear, since these assays have

279 been used in only a limited number of studies and applied to only a very

280 limited number of samples [33-37]. The utility of such assays remains

281 unknown as the range of subtypes they detect is unclear.

282

283 The first diagnostic PCR for Blastocystis was introduced in 2006 [25] but it

284 was later suspected to exhibit preferential amplification of some subtypes over

285 others. Since then, three diagnostic real-time PCR assays have been

286 reported. A real-time PCR based on an unknown Blastocystis target using

287 FRET probes was validated against ST1, ST3, and ST4 [38]. A SYBR green

288 real-time PCR used the SSU rRNA gene for detection of Blastocystis-specific

289 DNA (ST1-ST9), and subsequent subtyping was performed by melting curve

290 analysis [26]. The relatively large PCR product used (320 to $342 \mathrm{bp}$,

291 depending on the subtype) may impair the sensitivity of this test-especially

292 when DNA quality is not optimal-and the specificity of the assay was $95 \%$. 
293 The third real-time assay, using a hydrolysis probe based on the SSU rRNA

294 gene, was characterized by $100 \%$ specificity and ability to detect all nine

295 subtypes identified in humans so far [32]. The use of real-time PCR in large-

296 scale surveys would assist in identifying whether the development of

297 symptoms is related to infection intensity by simple analysis of threshold cycle

$298\left(C_{t}\right)$ values for individual samples, as this enables quantitation of the amount

299 of Blastocystis-specific DNA present. The same DNA samples may also be

300 used for subtyping and MLST protocols, hence allowing the detection and

301 evaluation of genetic diversity as well as the simple presence of Blastocystis

302 [22]. Blastocystis has also been included as a diagnostic target in commercial

303 gastrointestinal pathogen diagnostic panels such as Feconomics $®$ (Salubris

304 Inc, Boston, USA), EasyScreen ${ }^{\mathrm{TM}}$ Enteric Parasite Detection Kit (Genetic

305 Signatures, Sydney, Australia), and NanoChip® (Savyon Diagnostics, Israel).

307 While the potency of DNA-based methods is evident, they do not allow the

308 evaluation of whether differences in morphotypes are important. Several

309 different forms of Blastocystis have been described, including the avacuolar,

310 vacuolar, multivacuolar, granular, ameboid, and cyst stages. Although there

311 are a few reports of ameboid stages being detected only in symptomatic

312 Blastocystis carriers [eg. [39]), there is no consensus regarding the

313 significance of the different forms. Moreover, as mentioned earlier, it is not

314 clear whether some of these forms represent life-cycle stages, or are artifacts

315 resulting from exposure to oxygen or other stresses [2]. Relatively few studies

316 on the cyst stage are available [40-42], which is remarkable given that this is 
317 the stage that allows survival of the parasite in the environment and

318 transmission to a new host.

320 The high sensitivity of qualitative PCR for detection of Blastocystis DNA in

321 stool was reinforced by a recent study of Blastocystis in Senegalese children

322 [43], where the prevalence of Blastocystis among 93 children with and without 323 gastrointestinal symptoms was $100 \%$. When prevalence is so high there will

324 be little incentive for including Blastocystis PCR as a screening tool in the 325 clinical microbiology laboratory. However, where treatment of a patient with

326 Blastocystis has been undertaken, PCR methods are useful in post-treatment 327 follow-up to evaluate treatment efficacy.

329 This leads to one of the fundamental questions for clinical microbiology labs:

330 When is testing for Blastocystis appropriate? Data currently emerging indicate 331 that Blastocystis can be more common in individuals with a healthy GI system 332 than in patients with organic and functional bowel diseases (see below).

333 Therefore, the inclusion of Blastocystis as a specific target in screening 334 panels, alongside known pathogens such as Giardia, Cryptosporidium, and 335 Entamoeba histolytica, currently appears to make little sense in the clinical 336 microbiology laboratory. The presence of Blastocystis in stool samples most 337 likely implies that the carrier has been exposed to fecal-oral contamination, 338 which should prompt the laboratory to look more closely for the presence of 339 pathogens transmitted in the same way. However, since Blastocystis may 340 colonize the human colon for more than 10 years [44], it may be impossible to 341 identify when this contamination happened. This has important implications 
342 for the interpretation of clinical microbiology lab results. Blastocystis is

343 sometimes detected in stool samples of patients with diarrhea or other

344 gastrointestinal symptoms and in the absence of proven pathogens, so

345 clinicians might conclude that Blastocystis could be the cause of the

346 symptoms. If it is known that the infection is recent, the organism could

347 certainly be viewed as a potential cause of the symptoms; however, in most

348 cases it will be impossible to rule out that it has been present in the gut for

349 months - even years - and therefore is an incidental finding.

351 Another dilemma is the question of whether or not to report the presence of

352 Blastocystis in stool samples given that it is so common. Several studies have 353 sought to address this by setting a threshold number of Blastocystis

354 organisms detected microscopically per visual field at a specified

355 magnification before scoring the sample as positive; usually this has been set

356 at 5 organisms per 40x field (see references in [5]). However, the rationale for

357 this is unclear. It is known that shedding of both trophic and cyst forms of the 358 organism is irregular [45]. Moreover, several factors may influence the 359 number of organisms seen per visual field, including whether or not the 360 sample was fresh or preserved prior to analysis, and if preserved whether or 361 not the sample was fresh at the time of fixation. Real-time PCR would be 362 more sensitive and less affected by some of these variables. 363

364 In the event that symptoms are eventually linked to specific subtypes, 365 including those individual subtypes as specific targets in diagnostic panels 366 would be more relevant than including a general target for Blastocystis. 
Subtype-specific PCRs already exist, and barcoding of Blastocystis DNA amplified by generic primers can also be performed $[46,47]$. To date, diagnostic PCR methods have been developed and validated only for human clinical samples; no validated PCR method for detecting Blastocystis in environmental samples is yet available to the knowledge of the authors.

Given the extensive cryptic genetic diversity of Blastocystis [10, 15, 48], a number of tools have been developed to map its molecular epidemiology. Among these tools, two in particular have been widely used. A PCR assay for detecting subtypes using sequence-tagged-site (STS) primers was developed and refined in the early 1990s [49]. This approach involves the use of seven PCR reactions, one for each of subtypes $1-7$, and should be viewed as comprising a diagnostic method for each of these subtypes, circumventing the need for sequencing. The other method involves analysis of SSU rDNA variation. This approach has been developed independently by several groups, each of which used different regions of the SSU rRNA gene as markers [24-25, 50-56]. The barcoding method mentioned above, developed in 2006 by Scicluna et al., is one such example [46]. A comparison of the STS method and barcoding showed that barcoding should be preferred where possible for a variety of reasons [47]. First and foremost, barcoding enables the detection of subtypes beyond STs $1-7$ and further scrutiny of genetic diversity. The barcode region has also been validated as a marker of overall genetic diversity of Blastocystis [15]. 
391 Barcoding uses the primers RD5 and BhRDr, which amplify 600 bp at the 5'392 end of the SSU rRNA gene. Comparison of phylogenetic trees obtained by 393 analysis of barcoding sequences with those obtained using concatenated 394 sequences obtained by MLST (reflecting loci in the genome of the 395 mitochondrion-like organelle) demonstrated the appropriateness of using the 396 barcode region as a surrogate marker for overall genome diversity in this 397 particular organism [15]. The drawbacks of barcoding compared to the STS 398 method are that sequencing is required and that mixed subtype infections 399 may not always be evident in sequence chromatograms, and, even if they are, 400 they may prove difficult to decipher [47]. On the other hand, barcoding 401 enables more subtle analyses, namely SSU rDNA allele analysis [15]. A 402 public database is available (http://pubmlst.org/blastocystis/) that includes a 403 sequence repository for barcode sequences and those obtained by MLST. It 404 also has a BLAST facility, where individual or bulk fasta files can be uploaded 405 and analyzed for rapid identification of subtype and allele number, hence 406 eliminating the need for phylogenetic analysis. To date, 35 SSU rDNA alleles 407 within ST3 have been identified, whereas the number of SSU rDNA alleles for 408 ST4 and some other subtypes remains much more limited. However, some of 409 the allelic variation included is the result of sequencing of cloned DNA;

410 intragenomic SSU rDNA polymorphism has been reported [57, 58], and such 411 polymorphism will likely go unnoticed when sequences obtained directly from 412 PCR products are studied.

414 There is no doubt that DNA-based methods now enable us to carry out large 415 and well-designed research studies that are dependent on accurate detection 
416 and molecular characterization of Blastocystis. Such studies are required to

417 produce data that can shed light on the role of this organism in human health

418 and disease with a view to potentially developing diagnostics, biomarkers, and

419 therapies, including antimicrobial or probiotic agents, as appropriate.

5. CLINICAL SIGNIFICANCE AND EPIDEMIOLOGY

423 Even after more than 100 years, the role of Blastocystis in human health and

424 disease remains obscure. While Blastocystis has been speculated to be

425 involved in a range of organic and functional bowel diseases, it is clear that

426 asymptomatic carriage is common. This does not mean that Blastocystis does

427 not cause disease. The situation may resemble that for Giardia, where many

428 infections are asymptomatic (for example [59]), and Entamoeba histolytica,

429 where the proportion of symptomatic infections is at most $10 \%$ [60]. Case

430 reports and surveys continue to be published with regularity, mostly indicating

431 a link between Blastocystis and symptoms, although not always. We do not

432 propose to evaluate all the evidence here. However we do wish to highlight

433 two common issues: 1. Identification of an appropriate control group for

434 survey studies can be problematic; and 2. Excluding all other possible

435 etiologic agents or non-infectious causes of intestinal symptoms is almost

436 impossible.

438 While distinctive intestinal pathology has been clearly linked to the intestinal

439 protists Giardia, Cryptosporidium, and Entamoeba, there is little - if any -

440 evidence for direct pathology caused by Blastocystis. Phagocytosis of red 
441 blood cells is a well-known feature of Entamoeba histolytica that correlates

442 with virulence; there is only one study reporting phagocytosis in Blastocystis

443 [61]. No Blastocystis proteins such as glycoproteins or lectins that could

444 facilitate attachment to the gut epithelial layer have been identified, although

445 Denoeud et al. [57] have speculated that Blastocystis hydrolases might be

446 able to alter the colonic mucus layer (see below). It is generally accepted that

447 Blastocystis is non-invasive as well as lacking the ability to phagocytize the

448 microbiota or host-derived material.

450 When examining tissue sections from pig intestines, Fayer et al. [62] found

451 Blastocystis primarily in the lumen, usually associated with digested food

452 debris, and although sometimes in close proximity to or appearing to adhere

453 to the epithelium, there were no cells penetrating to the epithelium or the

454 lamina propria. These observations were confirmed by Wang et al. [63], who

455 did not observe any obvious pathology in histological sections of porcine gut

456 mucosal biopsies. In the latter study, Blastocystis cells were observed as

457 vacuolar/granular forms found within luminal material or in close proximity to

458 epithelial cells, with no evidence of attachment or invasion. When Blastocystis

459 is observed adhering to the epithelium in histological preparations it should be

460 kept in mind that histological procedures are likely to dissolve and eliminate

461 the mucus layer that is potentially separating Blastocystis from the mucosa in 462 vivo.

463

464 Despite the absence of invasion, discrete non-specific colonic inflammation

465 has been reported in a patient with both urticaria and what was characterized 
466

as 'heavy Blastocystis colonization'; Blastocystis eradication resulted in symptom resolution [64]. There are also some reports of Blastocystis having been found extra-intestinally, but in those cases it has not been possible to rule out that the presence of Blastocystis at these sites was merely a result of incidental or secondary colonization resulting from damage generated by other microorganisms or anatomical anomalies [65-68].

Blastocystis is one of several organisms to have been linked to Irritable Bowel Syndrome (IBS), including post-infectious IBS [69-71]. Genome analysis by Poirier et al. [72] identified various genes encoding hydrolases and serine and cysteine proteases, and the authors speculated that these potential virulence factors could be triggers of IBS by alteration of the mucus layer and interaction with tight junctions.

\section{Cross-sectional studies testing the hypothesis that Blastocystis is linked to} IBS mostly assume that, if the organism is associated with the disease, it should be more common in patients with IBS symptoms. The outcomes of such studies have been mixed, with some finding a higher prevalence of Blastocystis in IBS patients and some finding no difference or even lower prevalence (summarized in [12]). A few have looked at the subtype distribution, but although they have generally found differences between IBS and non-IBS patients, there is no consistency regarding the subtypes associated with IBS (summarized in [12]). IBS itself presents a diverse picture, with patients having diarrhea, constipation or a mixture of symptoms 
490 [69]. Even fewer investigations have been performed to look at potential links

491 between Blastocystis and subgroups within IBS.

493 IBS patients are likely to have multiple tests performed before a diagnosis is 494 made and, because of this, a common finding may well be Blastocystis in the 495 stool, which might then be suspected of being the agent responsible for the symptoms if no other candidates have been uncovered. So Blastocystis may be more commonly detected in IBS patients simply because the investigations are more thorough. Post-infectious IBS - a term describing the development of

499 IBS following treatment of an infection with antimicrobials [71] - adds another

500 complication, as the actual trigger for IBS may have been eliminated by

501 antimicrobial treatment, leaving Blastocystis behind to take the blame. It is 502 also impossible to exclude that Blastocystis was the initial trigger of IBS even

503 if it is no longer present. The potential links, if any, between Blastocystis and 504 IBS may be impossible to prove or disprove without large longitudinal cohort 505 studies.

506

507 One of the most interesting recent findings is that Blastocystis could be a 508 marker of gastrointestinal health rather than a cause of disease. This may in 509 fact not be surprising, given that we have been unable to reach a consensus 510 on a role for the organism in disease despite the large number and wide 511 range of investigations undertaken. A recent study identified Blastocystis as a 512 common member of the healthy human gut microbiota, with greater than $50 \%$ 513 of the healthy background population colonized [44]. Moreover, long-term 514 colonization trends were also noted; the same strains were present in the 
515 same hosts for up to 10 years [44]. A lower prevalence of Blastocystis in IBS

516 patients $(n=189)$ compared with healthy controls $(n=297), 14.5 \%$ versus

$51722 \%$ respectively $(p=0.09)$, was also highlighted in a recent study [73]; the

518 prevalence of Dientamoeba fragilis also differed significantly between the two

519 groups, with $D$. fragilis being similarly more common in individuals without

520 gastrointestinal symptoms. Another study, this time involving 96 healthy

521 controls and 100 patients with Inflammatory Bowel Disease (IBD) - a disease

522 affecting about 12,000 individuals in Denmark alone, $0.2 \%$ of the population -

523 detected a significantly lower prevalence of Blastocystis in IBD patients

524 compared with healthy controls $(p<0.05)$, with only $5 / 100$ IBD patients being

525 colonized by Blastocystis compared with 18/96 controls [74-75]. Interestingly,

526 four of the five positive IBD patients were in an inactive stage of the disease;

527 only $1 / 42$ patients with active IBD was a carrier.

529 Whether it is linked to gastrointestinal health or disease, it is clear that

530 Blastocystis is much more common than previously reported, reaching a

531 prevalence of $100 \%$ in some cohorts [43]. Individuals in communities with high

532 prevalence may become and remain infected from a very young age, while in

533 other communities, particularly where the overall prevalence is low, many

534 individuals may acquire Blastocystis later in life. For now, it is uncertain

535 whether the age at colonization - including whether Blastocystis becomes a

536 stable member of the intestinal microbiota from early on - is of any clinical

537 importance. It could be that in some regions of the world, Blastocystis might

538 be an 'emerging pathogen'. 
540 While recent observations suggest that Blastocystis colonization may be

541 inversely correlated with intestinal disease [44], we now know that the

542 bacterial component of the gut microbiota in IBS, IBD, and other intestinal

543 diseases is significantly different to that of the healthy human gut $[69,76]$.

544 Importantly, this may in fact indicate that Blastocystis is dependent on other

545 components of the microbiota to colonize and maintain a stable colonization in

546 the human gut. To test this prediction, we recently obtained access to data

547 from the MetaHIT Consortium (http://www.metahit.eu/), originally generated to

548 identify associations between intestinal bacterial communities and disease

549 patterns, including obesity, diabetes, and IBD [77]. From the data, we were

550 able to extract Blastocystis-specific DNA signatures, which enabled us to (1)

551 identify the relative prevalence of Blastocystis in each of the study groups,

552 and (2) to perform a preliminary investigation of the association between

553 Blastocystis and bacterial communities, in this case the so-called

554 'enterotypes' [77]. Our analysis [78] showed that: 1) Blastocystis was indeed

555 negatively associated with disease and absent in all 13 patients with Crohn's

556 disease (although not all studies have found this; [79]); and 2) very

557 intriguingly, Blastocystis was negatively associated with the Bacteroides

558 enterotype $(p<0.0001$, unpublished data). This finding may be linked to the

559 fact that the Bacteroides enterotype-compared with the Prevotella and the

560 Ruminococcus enterotypes - is characterized by low microbial diversity, and

561 this could therefore indicate that Blastocystis requires high overall microbial

562 diversity to become established in the human colon. However, it could also be

563 that some other unknown feature(s) of the enterotype may be responsible for

564 determining Blastocystis colonization, such as bacterial metabolic by- 
565 products. There is no doubt that studies of Blastocystis in the context of

566 intestinal bacterial communities and host physiology and immunity are likely to

567 advance our understanding of the clinical significance of Blastocystis. The

568 apparent impact of the gut flora on Blastocystis colonization may also mean

569 that standard animal models may be of limited use in exploring the effects of

570 Blastocystis on the human gut.

571

572 Comparing both bacterial and eukaryotic microbial communities in samples

573 from 23 individuals from agrarian communities in Malawi following traditional

574 lifestyles and from 13 individuals residing in Pennsylvania and Colorado,

575 USA, following a modern lifestyle, Parfrey et al. [80] recently showed that the

576 Malawi population harbored a diverse community of protists, including

577 Blastocystis, when compared to the North American populations, and that the

578 overall organismal diversity in the Malawian human gut is comparable to that

579 in other mammals. These, and other, data could indicate that the declining

580 diversity of the human bacterial microbiota identified in the West compared

581 with populations with traditional agrarian lifestyle has led to a reduced

582 prevalence of Blastocystis in Western populations [81].

583

584 It is also clear that geographical differences in subtype distributions may result

585 in geographical differences in the clinical significance of the parasite. There is 586 precedent in Entamoeba for cryptic genetic differences underlying differences

587 in the clinical outcome of infection (the E. histolytica/E. dispar story; [60]). So

588 a working hypothesis over the past few years has been that differences

589 between the clinical outcome of Blastocystis infection may reflect genetic 
590 differences in the organism. Hence, dozens of studies from all over the world

591 have sought to identify Blastocystis STs in both healthy and symptomatic

592 individuals (summarized in [11]). The distribution of subtypes across the major

593 geographical regions is depicted in Figure 3. So far, no particular subtype has

594 been linked consistently to disease. However, such a finding might not be

595 unexpected if the distribution of subtypes is uneven. While ST1, ST2, and ST3

596 appear to have a global distribution, current data suggest that ST4 is confined

597 mainly to Europe. ST4 was the only subtype identified in Danish patients with

598 acute diarrhea, but the overall prevalence of the parasite was also lower in

599 this group of patients than in others that have been studied in Denmark [82].

600 ST4 also dominated in symptomatic patients in Spain [83].

601

602 A significant gap in clinical Blastocystis research is the lack of large

603 randomized controlled clinical treatment trials [84-87]. To date these have

604 produced inconsistent and indeed contradictory results. It appears that no

605 single drug or drug combination currently in use consistently results in reliable

606 Blastocystis eradication [88-90]. Metronidazole has traditionally been used to

607 treat anaerobic microorganisms, including Entamoeba and Giardia; however,

608 its effect on Blastocystis has in some studies been minimal, with an

609 eradication rate as low as $0 \%$. Even the use of combinations such as

610 diloxanide furoate, secnidazole, and trimethoprim/sulfamethoxazole or

611 nitazoxanide may not result in consistent eradication [90].

612

613

614 6. GENOMICS 
616 With the advances in sequencing technology in recent years it has become

617 possible to sequence eukaryotic genomes quickly and relatively inexpensively

618 compared with even a few years ago. Perhaps surprisingly, the published

619 Blastocystis nuclear genome sequences at the time of writing are for ST7,

620 obtained by 'traditional' Sanger sequencing [57], and ST4, obtained by next

621 generation sequencing [91]. Others have not yet appeared in print despite

622 anecdotal evidence that suggests a flood of new data is about to arrive.

624 However, Blastocystis has two genomes. In addition to the nuclear genome it 625 also contains an organelle genome. In contrast to most anaerobic eukaryotes, 626 Blastocystis has mitochondrion-like organelles that have a quite normal 627 appearance under the transmission electron microscope (see [4]). It was

628 known for many years that these organelles contained DNA, based on 629 staining properties, but it was not until 2007 that the coding potential of these 630 molecules was uncovered. Two groups published sequences of the genomes 631 present in the mitochondrion-like organelle in three subtypes - STs 1, 4 and 7 632 [92-93]. The gene content and gene order of the 27-29 kilobasepair circular 633 molecules was identical, although the sequence divergence was

634 considerable. Subsequently, mitochondrion-like organelle genomes from

635 additional subtypes have been obtained (unpublished data) and these initial 636 observations have been upheld.

638 The gene content of the genome of the mitochondrion-like organelle is distinct 639 from the more familiar ones from mammals and yeast. Particularly notable is 
640 the absence of any genes encoding cytochrome and ATPase subunits and

641 the presence of a number of ribosomal protein genes. In common are the

642 genes encoding ribosomal RNAs and several tRNAs plus NADH

643 dehydrogenase (Complex I) subunits. The nuclear genomes and expressed

644 sequence tag (EST) surveys that are available confirm that the Blastocystis

645 mitochondrion-like organelle has only retained complexes I and II of the

646 electron transport chain, a characteristic shared with certain other anaerobic

647 eukaryotes. However, many other features of mitochondrial metabolism are

648 also present $[31,57]$. This is in contrast to the situation in, for example,

649 Giardia and Entamoeba where the genome has been lost completely and the

650 function of the resulting organelles (known as mitosomes) has become highly

651 reduced. Whether the Blastocystis organelle would follow a similar path given

652 enough time is impossible to predict.

653

654 The only published nuclear genomes at this time are for ST4 and ST7.

655 However, a recently published report on polyadenylation in Blastocystis also

656 includes data on a ST1 genome, suggesting its publication is imminent. The

657 polyadenylation report uncovered a unique situation in Blastocystis, where

658 around $15 \%$ of the stop codons in messenger RNAs are created through the

659 cleavage of a precursor and addition of the poly A tail to the mRNA [94]. This

660 is unprecedented outside of mitochondria. Given the degree of genetic

661 divergence between subtypes, comparative genomics may well reveal

662 significant differences between features of their nuclear genomes as well as

663 confirming genus-wide peculiarities, as in this case. 
665 Overall, the Blastocystis nuclear genome is quite small (under $19 \mathrm{Mb}$ ) with 666 relatively few genes (just over 6,000 ), quite a few of which appear to have 667 been acquired by horizontal gene transfer. Introns are numerous and small, 668 but repetitive DNA is rare. Of note is the fact that individual ribosomal RNA 669 cistrons are sometimes present in subtelomeric regions of the genome rather 670 than being exclusively found in long tandem arrays as in many other 671 eukaryotes [57].

\section{CONCLUSION}

Blastocystis is one of the most successful intestinal eukaryotes identified to date, being able to infect a wide range of host species. It may reside in the gut

677 for years on end and appears to show remarkably little susceptibility to

678 standard chemotherapeutic interventions, although analysis of biochemical

679 pathways identified through genome sequencing may generate some new

680 directions for drug interventions. However, the recognition of a high 681 prevalence of Blastocystis in healthy populations, identified using sensitive 682 molecular diagnostic tools, has heralded a paradigm shift in clinical 683 Blastocystis research. Studies of the gut microbiota in people with and without 684 Blastocystis are likely to provide valuable - if not critical - information to help 685 determine the role of Blastocystis in human health and disease.

8. ACKNOWLEDGEMENTS

688 Christen Rune Stensvold's work is partly funded by Marie Curie Actions (CIG 689 Project no. PCIG11-GA-2012-321614). 
693 [1] A. Alexieff, Sur la nature des formations dites "Kystes de Trichomonas intestinalis", C. R. Soc. Biol. 71 (1911) 296-298.

695 [2] A.A. Vdovenko, Blastocystis hominis: origin and significance of vacuolar and granular forms, Parasitol. Res. 86 (2000) 8-10.

697 [3] C.H. Zierdt, Blastocystis hominis: past and future, Clin. Microbiol. Rev. 4 (1991) 61-79.

699 [4] D.J. Stenzel, P.F. Boreham, Blastocystis hominis revisited, Clin. Microbiol. Rev. 9 (1996) 563-584

[5] K.S.W. Tan, New insights on classification, identification, and clinical relevance of Blastocystis spp., Clin. Microbiol. Rev. 21 (2008) 639665.

704 J6] J.D. Silberman, M.L. Sogin, D.D. Leipe, C.G. Clark, Human parasite 705 finds taxonomic home, Nature 380 (1996) 398.

706 [7] S.M. Adl, A.G. Simpson, C.E. Lane, J. Lukeš, D. Bass, S.S. Bowser, 707 M.W. Brown, F. Burki, M. Dunthorn, V. Hampl, A. Heiss, M. Hoppenrath, E. Lara, L. Le Gall, D.H. Lynn, H. McManus, E.A. Mitchell, S.E. Mozley-Stanridge, L.W. Parfrey, J. Pawlowski, S. Rueckert, R.S. Shadwick, C.L. Schoch, A. Smirnov, F.W. Spiegel, The revised classification of eukaryotes, J. Eukaryot. Microbiol. 59 (2012) 429-493. 
713 [8] M Kostka, I Čepička, V Hampl, J Flegr, Phylogenetic position of Karotomorpha and paraphyly of Proteromonadidae, Mol. Phylogenet. Evol. 43 (2007) 1167-1170.

716

[9] C.R. Stensvold, G.K. Suresh, K.S.W. Tan, R.C.A. Thompson, R.J. Traub, E. Viscogliosi, H. Yoshikawa, C.G. Clark, Terminology for Blastocystis subtypes: a consensus, Trends Parasitol. 23 (2007) 9396.

[10] M.A. Alfellani, D. Taner-Mulla, A.S. Jacob, C.A. Imeede, H. Yoshikawa, C.R. Stensvold, C.G. Clark, Genetic diversity of Blastocystis in livestock and zoo animals, Protist 164 (2013) 497-509.

[11] C.G. Clark, M. van der Giezen, M.A. Alfellani, C.R. Stensvold, Recent developments in Blastocystis research, Adv. Parasitol. 82 (2013) 1-32.

[12] M.A. Alfellani, C.R. Stensvold, A. Vidal-Lapiedra, E.S.U. Onuoha, A.F. Fagbenro-Beyioku, C.G. Clark, Variable geographic distribution of Blastocystis subtypes and its potential implications, Acta Trop. 126 (2013) 11-18.

[13] M.A. Alfellani, A.S. Jacob, N. Ortíz Perea, R.C. Krecek, D. TanerMulla, J.J. Verweij, B. Levecke, E. Tannich, C.G. Clark, C.R. Stensvold, Diversity and distribution of Blastocystis sp. subtypes in non-human primates, Parasitology 140 (2013) 966-971.

[14] W. Wang, H. Owen, R.J. Traub, L. Cuttell, T. Inpankaew, H. BielefeldtOhmann, Molecular epidemiology of Blastocystis in pigs and their in- 
contact humans in Southeast Queensland, Australia, and Cambodia, Vet. Parasitol. 203 (2014) 264-269.

[15] C.R. Stensvold, M.A. Alfellani, C.G. Clark, Levels of genetic diversity vary dramatically between Blastocystis subtypes, Infect. Genet. Evol. $12(2011)$ 263-273.

[16] Z. Wu, H. Mirza, K.S.W. Tan, Intra-subtype variation in enteroadhesion accounts for differences in epithelial barrier disruption and is associated with metronidazole resistance in Blastocystis subtype 7, PLoS Negl. Trop. Dis. 8 (2014) e2885.

[17] C.R. Stensvold, M.A. Alfellani, S. Nørskov-Lauritsen, K. Prip, E.L. Victory, C. Maddox, H.V. Nielsen, C.G. Clark, Subtype distribution of Blastocystis isolates from synanthropic and zoo animals and identification of a new subtype, Int. J. Parasitol. 39 (2009) 473-479.

[18] J.J. Verweij, C.R. Stensvold, Molecular testing for clinical diagnosis and epidemiological investigations of intestinal parasitic infections, Clin. Microbiol. Rev. 27 (2014) 371-418.

[19] C.H. Zierdt, W.S. Zierdt, B. Nagy, Enzyme-linked immunosorbent assay for detection of serum antibody to Blastocystis hominis in symptomatic infections, J. Parasitol. 81 (1995) 127-129.

[20] R. Nagel, R.J. Traub, M.M. Kwan, H. Bielefeldt-Ohmann, Blastocystis specific serum immunoglobulin in patients with irritable bowel 
syndrome (IBS) versus healthy controls, Parasit. Vectors. 8 (2015) 453.

[21] C.R. Stensvold, H.V. Nielsen, K. Mølbak, H.V. Smith, Pursuing the clinical significance of Blastocystis: diagnostic limitations, Trends Parasitol. 25 (2009) 23-29.

[22] C.R. Stensvold, Blastocystis: Genetic diversity and molecular methods for diagnosis and epidemiology, Trop. Parasitol. 3 (2013) 26-34.

[23] P.D. Scanlan, C.R. Stensvold, Blastocystis: getting to grips with our guileful guest, Trends Parasitol. 29 (2013) 523-529.

[24] C.R. Stensvold, M.C. Arendrup, C. Jespersgaard, K. Mølbak, H.V. Nielsen, Detecting Blastocystis using parasitologic and DNA-based methods: a comparative study, Diagn. Microbiol. Infect. Dis. 59 (2007) 303-307.

[25] C.R. Stensvold, A. Brillowska-Dabrowska, H.V. Nielsen, M.C. Arendrup, Detection of Blastocystis hominis in unpreserved stool specimens by using polymerase chain reaction, J. Parasitol. 92 (2006) $1081-1087$.

[26] P. Poirier, I. Wawrzyniak, A. Albert, H. El Alaoui, F. Delbac, V. Livrelli, Development and evaluation of a real-time PCR assay for detection and quantification of Blastocystis parasites in human stool samples: prospective study of patients with hematological malignancies, J. Clin. Microbiol. 49 (2011) 975-983. 
778 [27] T. Roberts, J. Barratt, J. Harkness, J. Ellis, D. Stark, Comparison of

779

780

781

782

783

784

785

786

787

788

789

790

791

792

793

794

795

796

797

798 microscopy, culture, and conventional polymerase chain reaction for detection of Blastocystis sp. in clinical stool samples, Am. J. Trop. Med. Hyg. 84 (2011) 308-312.

[28] Á.L. Londoño-Franco, J. Loaiza-Herrera, F.M. Lora-Suárez, J.E. Gómez-Marín, [Blastocystis sp. frequency and sources among children from 0 to 5 years of age attending public day care centers in Calarcá, Colombia (in Spanish)], Biomedica 34 (2014) 218-227.

[29] C.G. Clark, L.S. Diamond, Methods for cultivation of luminal parasitic protists of clinical importance, Clin. Microbiol. Rev. 15 (2002) 329-341.

[30] S. Leelayoova, P. Taamasri, R. Rangsin, T. Naaglor, U. Thathaisong, M. Mungthin, In-vitro cultivation: a sensitive method for detecting Blastocystis hominis, Ann. Trop. Med. Parasitol. 96 (2002)803-807.

[31] A. Stechmann, K. Hamblin, V. Pérez-Brocal, D. Gaston, G.S. Richmond, M. van der Giezen, C.G. Clark, A.J. Roger, Organelles in Blastocystis that blur the distinction between mitochondria and hydrogenosomes, Curr. Biol. 18 (2008) 580-585.

[32] C.R. Stensvold, U.N. Ahmed, L.O. Andersen, H.V. Nielsen, Development and evaluation of a genus-specific, probe-based, internal process controlled real-time PCR assay for sensitive and specific detection of Blastocystis, J. Clin. Microbiol. 50 (2012) 1847-1851. 
799

800

801

802

803

804

805

806

807

808

809

810

811

812

813

814

815

816

817

818

819
[33] R. Fayer, M. Santin, D. Macarisin, Detection of concurrent infection of dairy cattle with Blastocystis, Cryptosporidium, Giardia, and Enterocytozoon by molecular and microscopic methods, Parasitol. Res. 111 (2012) 1349-1355.

[34] F. Dogruman-Al, Z. Simsek, K. Boorom, E. Ekici, M. Sahin, C. Tuncer, S. Kustimur, A. Altinbas, Comparison of methods for detection of Blastocystis infection in routinely submitted stool samples, and also in IBS/IBD Patients in Ankara, Turkey. PLoS One 5 (2010) e15484.

[35] S.M. El-Marhoumy, K. Abd EL-Nouby, Z.S. Shoheib, A.M. Salama, Prevalence and diagnostic approach for a neglected protozoon Blastocystis hominis, Asian Pac. J. Trop. Med. 5 (2015) 51-59.

[36] R. Gould, K. Boorom, Blastocystis surface antigen is stable in chemically preserved stool samples for at least 1 year, Parasitol. Res. 112 (2013) 2469-2471.

[37] F. Dogruman-Al, S. Turk, G. Adiyaman-Korkmaz, A. Hananel, L. Levi, J. Kopelowitz, O. Babai, S. Gross, Z. Greenberg, Y. Herschkovitz, I. Mumcuoglu, A novel ELISA test for laboratory diagnosis of Blastocystis spp. in human stool specimens, Parasitol. Res. 114 (2015) 495-500.

[38] M.S. Jones, R.D. Ganac, G. Hiser, N.R. Hudson, A. Le, C.M. Whipps, Detection of Blastocystis from stool samples using real-time PCR, Parasitol. Res. 103 (2008) 551-557. 
820 [39] T.C. Tan, G.S. Kumar, Predominance of amoeboid forms of Blastocystis hominis in isolates from symptomatic patients, Parasitol. Res. 98 (2006) 189-193.

\section{3}

[40] D.J. Stenzel, P.F. Boreham, A cyst-like stage of Blastocystis hominis, Int. J. Parasitol. 21 (1991) 613-615.

825

826

[41] K.T. Moe, M. Singh, J. Howe, L.C. Ho, S.W. Tan, G.C. Ng, X.Q. Chen, E.H. Yap, Observations on the ultrastructure and viability of the cystic stage of Blastocystis hominis from human feces, Parasitol. Res. 82 (1996) 439-444.

[42] I.F. Abou El Naga, A.Y. Negm, Morphology, histochemistry and infectivity of Blastocystis hominis cyst, J. Egypt. Soc. Parasitol. 31

[43] D. El Safadi, L. Gaayeb, D. Meloni, A. Cian, P. Poirier, I. Wawrzyniak, F. Delbac, F. Dabboussi, L. Delhaes, M. Seck, M. Hamze, G. Riveau, E. Viscogliosi, Children of Senegal River Basin show the highest prevalence of Blastocystis sp. ever observed worldwide, BMC Infect.

[44] P.D. Scanlan, C.R. Stensvold, M. Rajilić-Stojanović, H.G. Heilig, W.M. De Vos, P.W. O'Toole, P.D. Cotter, The microbial eukaryote Blastocystis is a prevalent and diverse member of the healthy human gut microbiota, FEMS Microbiol. Ecol. 90 (2014) 326-330. 
842 [45] G.D. Vennila, G.S. Kumar, A.K. Anuar, S. Rajah, R. Saminathan, S. Sivanandan, K. Ramakrishnan, Irregular shedding of Blastocystis hominis, Parasitol. Res. 85 (1999) 162-164.

845 [46] S.M. Scicluna, B. Tawari, C.G. Clark, DNA barcoding of Blastocystis, 846 Protist 157 (2006) 77-85.

847

848

849

850

851

852

853

854

855

856

857

858

859

860

861

862

[47] C.R .Stensvold, Comparison of sequencing (barcode region) and sequence-tagged-site PCR for Blastocystis subtyping, J. Clin. Microbiol. 51 (2013) 190-194.

[48] C.G. Clark, Extensive genetic diversity in Blastocystis hominis, Mol. Biochem. Parasitol. 87 (1997) 79-83.

[49] H. Yoshikawa, Z. Wu, I. Kimata, M. Iseki, I.K.M. Ali, M.B. Hossain, V. Zaman, R. Haque, Y. Takahashi, Polymerase chain reaction-based genotype classification among human Blastocystis hominis populations isolated from different countries, Parasitol. Res. 92 (2004) 22-29.

[50] C.R .Stensvold, M. Lebbad, J.J. Verweij, C. Jespersgaard, G. von Samson-Himmelstjerna, S.S. Nielsen, H.V. Nielsen, Identification and delineation of members of the Entamoeba complex by pyrosequencing, Mol. Cell. Probes 24 (2010) 403-406.

[51] M. Özyurt, Ö. Kurt, K. Mølbak, H.V. Nielsen, T. Haznedaroglu, C.R Stensvold, Molecular epidemiology of Blastocystis infections in Turkey, Parasitol. Int. 57 (2008) 300-306. 
863

864

865

866

867

868

869

870

871

872

873

874

875

876

877

878

879

880

881

882

883

884

885

[52] C.R .Stensvold, H.C. Lewis, H.M. Hammerum, L.J. Porsbo, S.S.

Nielsen, K.E. Olsen, M.C. Arendrup, H.V. Nielsen, K. Mølbak K, Blastocystis: unravelling potential risk factors and clinical significance of a common but neglected parasite, Epidemiol. Infect. 137 (2009) 1655-1663.

[53] U. Parkar, R.J. Traub, S. Kumar, M. Mungthin, S. Vitali, S. Leelayoova, K. Morris, R.C.A. Thompson, Direct characterization of Blastocystis from faeces by PCR and evidence of zoonotic potential, Parasitology 134 (2007) 359-367.

[54] U. Parkar, R.J. Traub, S. Vitali, A. Elliot, B. Levecke, I. Robertson, T. Geurden, J. Steele, B. Drake, R.C.A. Thompson, Molecular characterization of Blastocystis isolates from zoo animals and their animal-keepers, Vet. Parasitol. 169 (2010) 8-17.

[55] M. Santín, M.T. Gómez-Muñoz, G. Solano-Aguilar, R. Fayer, Development of a new PCR protocol to detect and subtype Blastocystis spp. from humans and animals, Parasitol. Res. 109 (2011) 205-212.

[56] K.H. Wong, G.C. Ng, R.T. Lin, H. Yoshikawa, M.B. Taylor, K.S.W. Tan, Predominance of subtype 3 among Blastocystis isolates from a major hospital in Singapore, Parasitol. Res. 102 (2008) 663-670.

[57] F. Denoeud, M. Roussel, B. Noel, I. Wawrzyniak, C. Da Silva, M. Diogon, E. Viscogliosi, C. Brochier-Armanet, A. Couloux, J. Poulain, B. Segurens, V. Anthouard, C. Texier, N. Blot, P. Poirier, G.C. Ng, K.S.W. Tan, F. Artiguenave, O. Jaillon, J.M. Aury, F. Delbac, P. Wincker, C.P. 
886

887

888

889

890

891

892

893

894

895

896

897

898

899

900

901

902

903

904

905

906
Vivarès, $\mathrm{H}$. El Alaoui, Genome sequence of the stramenopile Blastocystis, a human anaerobic parasite, Genome Biol. 12 (2011) R29.

[58] D. Meloni, P. Poirier, C. Mantini, C. Noël, N. Gantois, I. Wawrzyniak, F. Delbac, M. Chabé, L. Delhaes, E. Dei-Cas, P.L. Fiori, H. El Alaoui, E. Viscogliosi, Mixed human intra- and inter-subtype infections with the parasite Blastocystis sp., Parasitol. Int. 61 (2012) 719-722.

[59] M.G. Tellevik, S.J. Moyo, B. Blomberg, T. Hjøllo, S.Y. Maselle, N. Langeland, K. Hanevik, Prevalence of Cryptosporidium parvum/hominis, Entamoeba histolytica and Giardia lamblia among young children with and without diarrhea in Dar es Salaam, Tanzania, PLoS Negl. Trop. Dis. 9 (2015) e0004125

[60] B.S. Pritt, C.G. Clark, Amebiasis, Mayo Clin. Proc. 83 (2008) 11541159.

[61] L.A. Dunn, P.F. Boreham, D.J. Stenzel, Ultrastructural variation of Blastocystis hominis stocks in culture, Int. J. Parasitol. 19 (1989) 43-56.

[62] R. Fayer, T. Elsasser, R. Gould, G. Solano, J. Urban Jr, M. Santin, Blastocystis tropism in the pig intestine, Parasitol. Res. 113 (2014) $1465-1472$ 
908 [63] W. Wang, H. Bielefeldt-Ohmann, R.J. Traub, L. Cuttell, H. Owen, 909 Location and pathogenic potential of Blastocystis in the porcine intestine, PLoS One. 9 (2014) e103962

912 [64] C. Vogelberg, C.R. Stensvold, S. Monecke, A. Ditzen, K. Stopsack, U. Heinrich-Gräfe, C. Pöhlmann, Blastocystis sp. subtype 2 detection during recurrence of gastrointestinal and urticarial symptoms, Parasitol. Int. 59 (2010) 469-471.

916

917 [65] H.L. Santos, F.C. Sodré, H.W. de Macedo, Blastocystis sp. in splenic 918 cysts: causative agent or accidental association? A unique case report, Parasit. Vectors. 7 (2014) 207.

921 [66] T.V. Prodeus, O.P. Zelia, T.A. Khlebnikova, D.A. Pikul', [Extraenteric infection caused by Blastocystis spp. in a female patient with liver abscess (in Russian)], Med. Parazitol. (Mosk.) Apr-Jun (2014) 6-9.

925 [67] W.D. Patino, D. Cavuoti, S.K. Banerjee, K. Swartz, R. Ashfaq, T. Gokaslan, Cytologic diagnosis of Blastocystis hominis in peritoneal fluid: a case report, Acta Cytol. 52 (2008) 718-720. 
929 [68] R. Silard, M. Petrovici, D. Panaitescu, V. Stoicescu, Blastocystis

930 hominis in the liver of Cricetus auratus, Arch. Roum. Pathol. Exp.

$931 \quad$ Microbiol. 36 (1977) 55-60.

932

933 [69] S.M. Collins, A role for the gut microbiota in IBS, Nat. Rev.

934 Gastroenterol. Hepatol. 11 (2014) 497-505.

935

936 [70] P.J. Kennedy, J.F. Cryan, T.G. Dinan, G. Clarke, Irritable bowel syndrome: A microbiome-gut-brain axis disorder?, World J.

938 Gastroenterol. 20 (2014)14105-14125.

939

940 [71] J.K. Beatty, A. Bhargava, A.G. Buret, Post-infectious irritable bowel syndrome: mechanistic insights into chronic disturbances following enteric infection, World J. Gastroenterol. 20 (2014) 3976-3985

944 [72] P. Poirier, I. Wawrzyniak, C.P. Vivarès, F. Delbac, H. El Alaoui, New 945 insights into Blastocystis spp.: a potential link with irritable bowel syndrome, PLoS Pathog. 8 (2012) e1002545. 
[73] L.R. Krogsgaard, A.L. Engsbro, C.R. Stensvold, H.V. Nielsen, P. Bytzer, The prevalence of intestinal parasites is not greater among individuals with Irritable Bowel Syndrome: a population-based casecontrol study, Clin Gastroenterol Hepatol. 13 (2014) 507-513.

[74] A.M. Petersen, H.V. Nielsen, C.R. Stensvold, J.H. Engberg, A. FriisMoller, I. Nordgaard-Lassen, S. Wildt, K.A. Krogfelt, Blastocystis and Dientamoeba fragilis in active and inactive Inflammatory Bowel Disease, Gastroenterology 140 (2011) S329-S330.

[75] A.M. Petersen, C.R. Stensvold, H. Mirsepasi, J. Engberg, A. FriisMøller, L.J. Porsbo, A.M. Hammerum, I. Nordgaard-Lassen, H.V. Nielsen, K.A. Krogfelt, Active ulcerative colitis associated with low prevalence of Blastocystis and Dientamoeba fragilis infection, Scand. J. Gastroenterol. 48 (2013) 638-639.

[76] A.D. Kostic, R.J. Xavier, D. Gevers, The microbiome in inflammatory bowel disease: current status and the future ahead, Gastroenterology. 146 (2014) 1489-1499.

[77] M. Arumugam, J. Raes, E. Pelletier, D. Le Paslier, T. Yamada, D.R. Mende, G.R. Fernandes, J. Tap, T. Bruls, J.M. Batto, M. Bertalan, N. Borruel, F. Casellas, L. Fernandez, L. Gautier, T. Hansen, M. Hattori, T. Hayashi, M. Kleerebezem, K. Kurokawa, M. Leclerc, F. Levenez, C. Manichanh, H.B. Nielsen, T. Nielsen, N. Pons, J. Poulain, J. Qin, T. Sicheritz-Ponten, S. Tims, D. Torrents, E. Ugarte, E.G. Zoetendal, J. Wang, F. Guarner, O. Pedersen, W.M. de Vos, S. Brunak, J. Doré, M. 

Antolín, F. Artiguenave, H.M. Blottiere, M. Almeida, C. Brechot, C. Cara, C. Chervaux, A. Cultrone, C. Delorme, G. Denariaz, R. Dervyn, K.U. Foerstner, C. Friss, M. van de Guchte, E. Guedon, F. Haimet, W. Huber, J. van Hylckama-Vlieg, A. Jamet, C. Juste, G. Kaci, J. Knol, O. Lakhdari, S. Layec, K. Le Roux, E. Maguin, A. Mérieux, R. Melo Minardi, C. M'rini, J. Muller, R. Oozeer, J. Parkhill, P. Renault, M. Rescigno, N. Sanchez, S. Sunagawa, A. Torrejon, K. Turner, G. Vandemeulebrouck, E. Varela, Y. Winogradsky, G. Zeller, MetaHIT Consortium, J. Weissenbach, S.D. Ehrlich, P. Bork, Enterotypes of the human gut microbiome, Nature 473 (2011) 174-180.

[78] L.O. Andersen, I. Bonde, H.B. Nielsen, C.R. Stensvold, A retrospective metagenomics approach to studying Blastocystis, FEMS Microbiol. Ecol. 91 (2015) fiv072.

[79] A.H. Cekin, Y. Cekin, Y. Adakan, E. Tasdemir, F.G. Koclar, B.O. Yolcular, Blastocystosis in patients with gastrointestinal symptoms: a case-control study, BMC Gastroenterol. 12 (2012) 122.

[80] L.W. Parfrey, W.A. Walters, C.L. Lauber, J.C. Clemente, D. BergLyons, C. Teiling, C. Kodira, M. Mohiuddin, J. Brunelle, M. Driscoll, N. Fierer, J.A. Gilbert, R. Knight, Communities of microbial eukaryotes in the mammalian gut within the context of environmental eukaryotic diversity, Front. Microbiol. 5 (2014) 298. 
992

[81] C. De Filippo, D. Cavalieri, M. Di Paola, M. Ramazzotti, J.B. Poullet, S. Massart, S. Collini, G. Pieraccini, P. Lionetti, Impact of diet in shaping gut microbiota revealed by a comparative study in children from Europe and rural Africa, Proc. Natl. Acad. Sci. USA. 107 (2010) 14691-14696.

[82] C.R. Stensvold, D.B. Christiansen, K.E. Olsen, H.V. Nielsen, Blastocystis sp. subtype 4 is common in Danish Blastocystis-positive patients presenting with acute diarrhea, Am. J. Trop. Med. Hyg. 84 (2011) 883-885.

[83] M.V. Domínguez-Márquez, R. Guna, C. Muñoz, M.T. Gómez-Muñoz, R. Borrás, High prevalence of subtype 4 among isolates of Blastocystis hominis from symptomatic patients of a health district of Valencia (Spain), Parasitol. Res. 105 (2009) 949-955.

[84] K. Heyland, M. Friedt, P. Buehr, C.P. Braegge, No advantage for antibiotic treatment over placebo in Blastocystis hominis-positive children with recurrent abdominal pain, J. Pediatr. Gastroenterol. Nutr. 54 (2012) 677-679.

[85] B. Speich, H. Marti, S.M. Ame, S.M. Ali, I.I. Bogoch, J. Utzinger, M. Albonico, J. Keiser, Prevalence of intestinal protozoa infection among school-aged children on Pemba Island, Tanzania, and effect of singledose albendazole, nitazoxanide and albendazole-nitazoxanide, Parasit. Vectors 6 (2013) 3. 
1013

1014

1015

1016

1017

1018

1019

1020

1021

1022

1023

1024

1025

1026

1027

1028

1029

1030

1031

1032

1033
[86] J.F. Rossignol, S.M. Kabil, M. Said, H. Samir, A.M. Younis, Effect of nitazoxanide in persistent diarrhea and enteritis associated with Blastocystis hominis, Clin. Gastroenterol. Hepatol. 3: (2005) 987-991.

[87] L. Nigro, L. Larocca, L. Massarelli, I. Patamia, S Minniti, F. Palermo, B. Cacopardo, A placebo-controlled treatment trial of Blastocystis hominis infection with metronidazole, J. Travel Med. 10 (2003) 128-130.

[88] C.R. Stensvold, H.V. Smith, R. Nagel, K.E. Olsen, R.J. Traub, Eradication of Blastocystis carriage with antimicrobials: reality or delusion?, J. Clin. Gastroenterol. 44 (2010) 85-90.

[89] T. Roberts, J. Ellis, J. Harkness, D. Marriott, D. Stark, Treatment failure in patients with chronic Blastocystis infection, J. Med. Microbiol. 63 (2014) 252-257.

[90] R. Nagel, H. Bielefeldt-Ohmann, R. Traub, Clinical pilot study: efficacy of triple antibiotic therapy in Blastocystis positive irritable bowel syndrome patients, Gut Pathog. 6 (2014) 34.

[91] I. Wawrzyniak, D. Courtine, M. Osman, C. Hubans-Pierlot, A. Cian, C. Nourrisson, M. Chabe, P. Poirier, A. Bart, V. Polonais, P. DelgadoViscogliosi, H. El Alaoui, A. Belkorchia, T. van Gool, K.S.W. Tan, S. Ferreira, E. Viscogliosi, F. Delbac, Draft genome sequence of the intestinal parasite Blastocystis subtype 4-isolate WR1, Genomics Data 4 (2015) 22-23. 
1034 [92] V. Pérez-Brocal, C.G. Clark, Analysis of two genomes from the

1035

1036

1037

1038

1039

1040

1041

1042

1043

1044

1045

1046 mitochondrion-like organelle of the intestinal parasite Blastocystis:

complete sequences, gene content and genome organization, Mol.

Biol. Evol. 25 (2008) 2475-2482.

[93] I. Wawrzyniak, M. Roussel, M. Diogon, A. Couloux, C. Texier, K.S.W.

Tan, C.P. Vivarès, F. Delbac, P. Wincker, H. El Alaoui, Complete

circular DNA in the mitochondria-like organelles of Blastocystis

hominis, Int. J. Parasitol. 38 (2008) 1377-1382.

[94] V. Klimeš, E. Gentekaki, A.J. Roger, M. Eliáš, A large number of nuclear genes in the human parasite Blastocystis require mRNA polyadenylation to create functional termination codons, Genome Biol. Evol. 6 (2014) 1956-1961. 
1047 Figure 1. Light microscopy images of Blastocystis. A. Blastocystis in culture.

1048 Using Robinson's and other media [29], Blastocystis often reaches high

1049 density in xenic culture. This stage is typically reported as 'vacuolar' due to

1050 the large central region of uncertain function. Organelles are seen as 'dots'

1051 along the periphery of the cell. B and C. Blastocystis in fecal smears, stained

1052 using iron-hematoxylin. Prominent nuclei are seen in the periphery of the cells

1053 as the most conspicuous morphological hallmark, along with the large central

1054 'void'. Other organelles can be discerned as smaller peripheral 'dots', which

1055 will include the mitochondrion-like organelles, etc. However, these can only be

1056 positively identified by transmission electron microscopy. Images courtesy of

1057 John Williams (A) and Claire Rogers (B, C), Diagnostic Parasitology

1058 Laboratory, London School of Hygiene and Tropical Medicine.

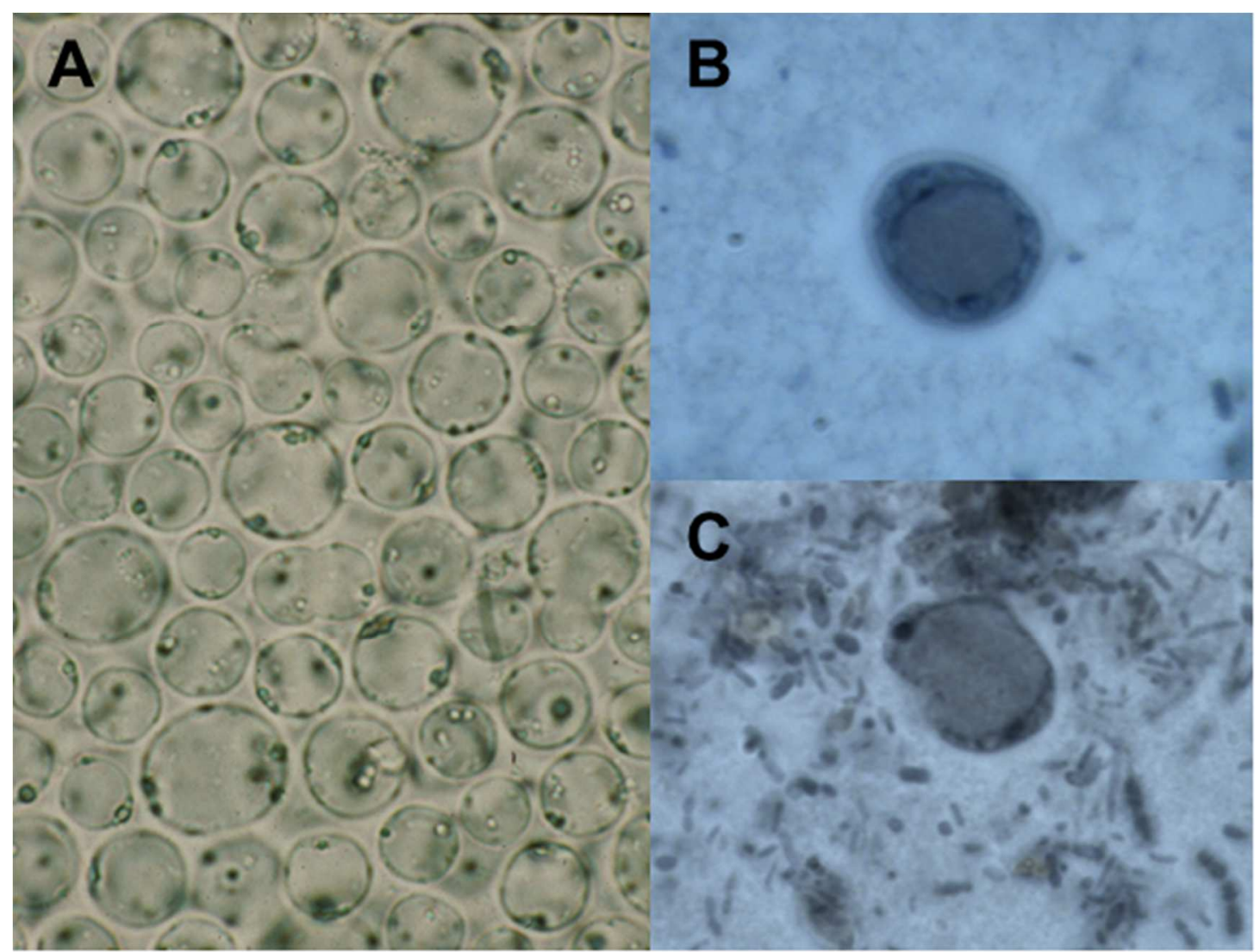


1061 Figure 2. Host range and relative prevalence of Blastocystis subtypes. In this

1062 schematic, the range of subtypes reported for four major host groups

1063 (humans, non-human primates, ungulates and birds) is shown. In the circle,

1064 the numbers are those of the most common subtypes found in the respective

1065 host, with the integer font size proportional to its prevalence. Numbers in the

1066 magnified boxes represent those subtypes that each constitute less than 5\%

1067 of the total samples subtyped to date. Derived from the numbers presented in

1068 reference [10]. As an indication, prevalence figures for STs 1-4 in humans are

$106928.0 \%, 10.9 \%, 44.4 \%$ and $10.0 \%$ respectively.

1070
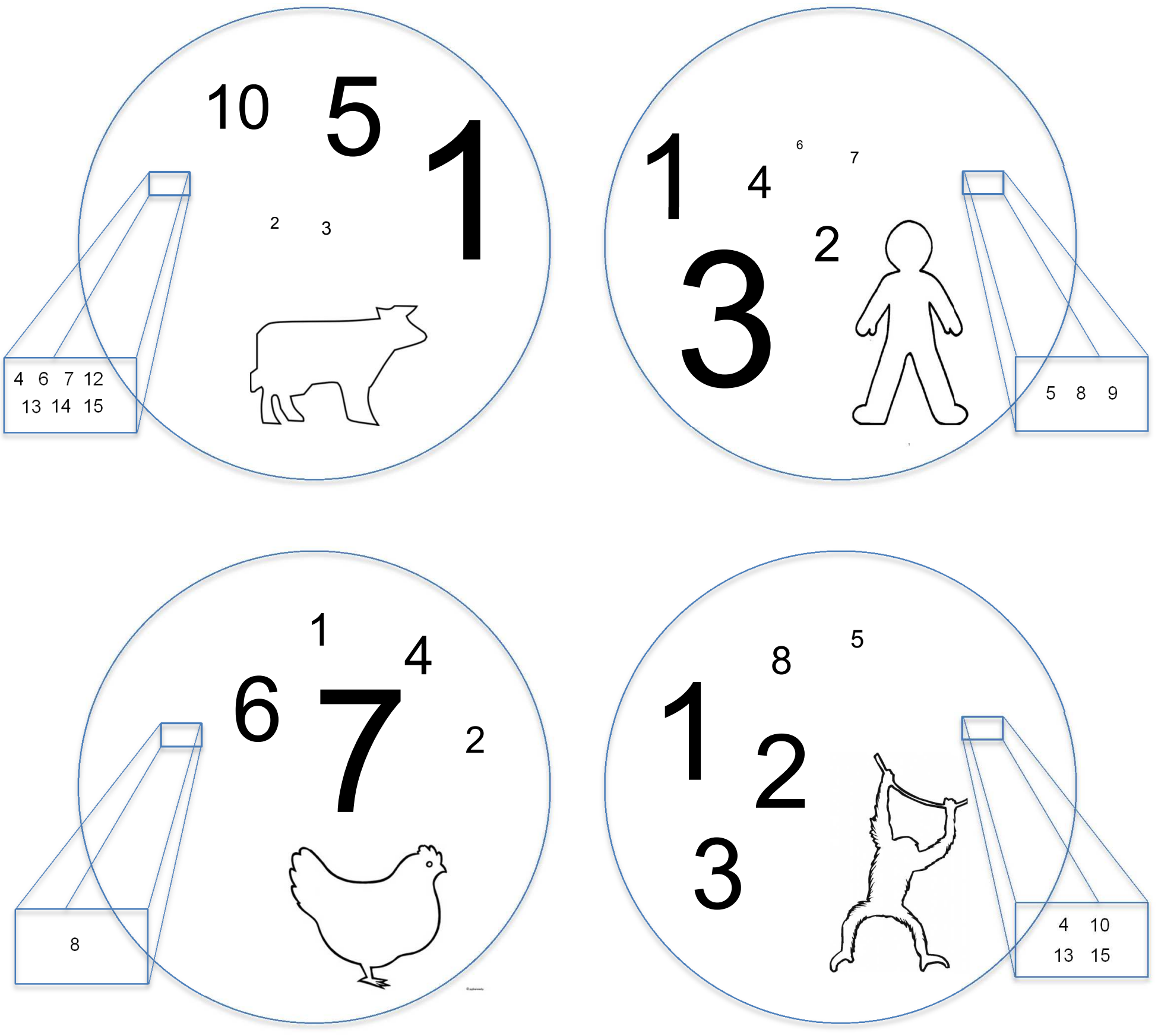
1071 Figure 3: Pie charts of human Blastocystis subtype distributions in Europe (A) 1072 and the rest of the world $(B)$. These were produced from the data presented in 1073 Alfellani et al. [12]. Of note is the fact that although ST4 accounted for $10 \%$ of 1074 the samples across the world $(N=318), 87 \%$ of these $(278)$ were from 1075 Europe, suggesting that ST4 is more or less geographically restricted to 1076 Europe.
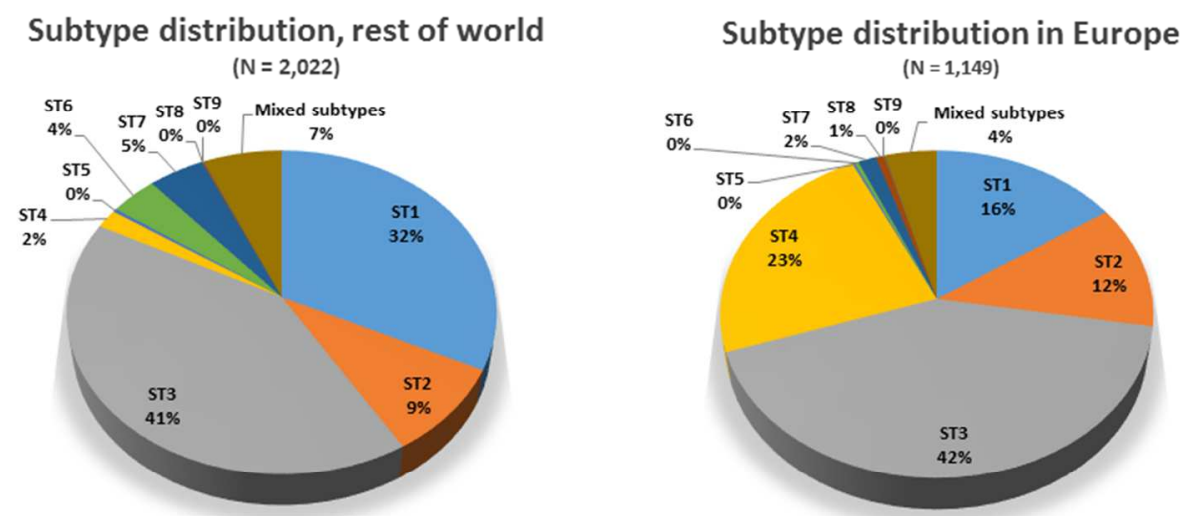